\title{
Correction: The acetyltransferase GCN5 maintains ATRA-resistance in non-APL AML
}

Melanie Kahl - Annamaria Brioli • Martin Bens 10 - Florian Perner • Anne Kresinsky • Ulf Schnetzke - Anna Hinze • Yordan Sbirkov · Sven Stengel · Giorgia Simonetti - Giovanni Martinelli · Kevin Petrie · Arthur Zelent •

Frank-Dietmar Böhmer - Marco Groth (1) - Thomas Ernst • Florian H. Heidel · Sebastian Scholl • Andreas Hochhaus • Tino Schenk

Published online: 16 January 2020

(c) The Author(s), under exclusive licence to Springer Nature Limited 2020

\section{Correction to: Leukemia}

https://doi.org/10.1038/s41375-019-0581-y

Since publication of the original article the authors noticed that affiliation ${ }^{8}$ Istituto Scientifico Romagnolo per lo Studio e la Cura dei Tumori (IRST) IRCCS, Meldola, Italy was presented incorrectly. This has now been corrected in the HTML and PDF versions of this article. 